\title{
Thermostring quantization. An interpretation of strings as particles at finite temperatures ${ }^{1}$
}

\author{
Zahid Zakir ${ }^{2}$
}

\begin{abstract}
In a space-temperature configurational manifold an instantaneous temperature path of a point particle can be represented as a string of length $\mathrm{L}=1 / \mathrm{kT}$ (thermostring). The thermostring swepts a surface in the space-time-temperature manifold at its temporal evolution. The thermostring is closed, its points can be rearranged and the charge is distributed along the length. Some predictions of this method for statistical mechanics and string theories are discussed.
\end{abstract}

PACS: 11.10.Wx, 11.10.Kk, 11.25.-w, 11.25.Uw, 11.25.Wx

Key words: quantization, finite temperature, extra dimensions, strings, branes

\section{Content}

Introduction 1

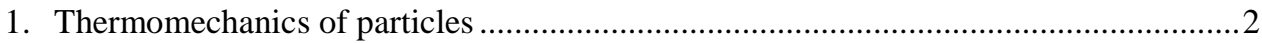



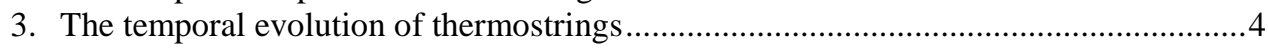

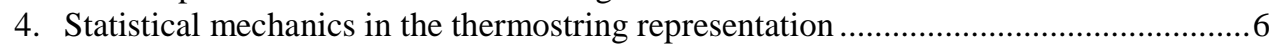

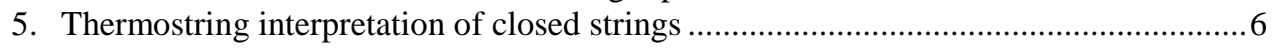

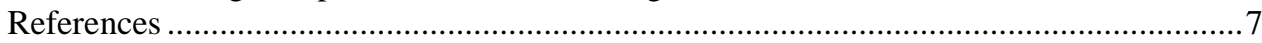

\section{Introduction}

A well known analogy between quantum mechanics and quantum statistical mechanics of particles under the replacements $\Delta t \rightarrow-i \Delta \beta, \Delta \beta \rightarrow i \Delta t$ has been commonly used as a formal procedure at the consideration of its $t$ - or $\beta$-evolutions [1]. Here $\Delta t$ is a temporal interval, $\Delta \beta$ is (inverse) temperature evolution parameter's interval ("cold"), $T$ is temperature, $k$ is the Boltzmann constant. However, if we consider $\beta$ evolution of particles in combination with $t$ evolution, this analogy leads to some $(t-\beta)$ symmetry. Here the inverse temperature parameter $\beta$ can be treated as a new geometrical degree of freedom in addition to the spatial and temporal ones. So, as a result, we have some space-time-temperature manifold with $D=d+2$ dimensions, where $d$ is space dimensionality. We shall introduce in this manifold the thermostring representation of density matrix or the thermostring quantization (see also [3-4]). For non-relativistic particles the thermostring quantization leads to some reformulation of the statistical mechanics. The thermostring quantization of relativistic particles at some invariant temperature $T_{0}$, corresponding to some invariant length, for example, to the Planck length or charge (gravitational) radii of particles, leads to the closed string formalism. Here the invariance of the temperature $T_{0}$ is very important and this fact

\footnotetext{
${ }^{1}$ The preprint of the paper has been presented in 1998 (revised 2003): Zakir Z. arXiv:hep-th/9809170

${ }^{2}$ Centre for Theoretical Physics and Astrophyics, Tashkent, Uzbekistan; zahidzakir@theor-phys.org
} 
allows us to treat the temperature degree of freedom as one of geometrical dimensions of the physical manifold for the given system. The thermostring quantization makes it possible a new physical interpretation of superstrings IIA and heterotic strings in terms of point particles..

\section{Thermomechanics of particles}

The density matrix $\rho$ for mixed states of a non-relativistic particle at the finite temperature $T$ can be represented as

$$
\begin{aligned}
\rho\left(\mathbf{r}, \mathbf{r}_{0} ; \Delta \beta\right) & =\sum_{i} \exp \left(-E_{i} \Delta \beta\right) \times \psi_{i}(\mathbf{r}) \psi_{i}^{*}\left(\mathbf{r}_{0}\right)= \\
& =\sum_{i} \psi_{i}(\mathbf{r}, \beta) \psi_{i}^{*}\left(\mathbf{r}_{0}, \beta_{0}\right)=\rho\left(\mathbf{r}, \beta ; \mathbf{r}_{0}, \beta_{0}\right),
\end{aligned}
$$

where $\Delta \beta=\beta-\beta_{0}=1 / k T, E_{i}$ - is the energy of particle, $\mathbf{r}$ is a spatial radius-vector. Here the wave functions $\psi_{i}(\mathbf{r}, \beta)$ describe pure states of the particle in a $(\mathbf{r}, \beta)$ manifold with the Hamiltonian $H$ :

$$
\begin{gathered}
\psi_{i}(\mathbf{r}, \beta)=\exp (-H \beta) \psi_{i}(\mathbf{r}), \\
\psi_{i}^{*}(\mathbf{r}, \beta)=\psi_{i}^{*}(\mathbf{r}) \exp (H \beta),
\end{gathered}
$$

normalized as:

$$
\int \psi_{i}^{*}(\mathbf{r}, \beta) \psi_{j}(\mathbf{r}, \beta) d \mathbf{r}=\delta_{i j} .
$$

In $(\mathbf{r}, \beta)-$ manifold, the density matrix $\rho$ plays the role of a transition amplitude for the $\beta$-evolution of the wave functions $\psi_{i}(\mathbf{r}, \beta)$ :

$$
\psi_{i}(\mathbf{r}, \beta)=\int d \mathbf{r}_{0} \rho\left(\mathbf{r}, \beta ; \mathbf{r}_{0}, \beta_{0}\right) \psi_{i}\left(\mathbf{r}_{0}, \beta_{0}\right),
$$

with the property:

$$
\rho\left(\mathbf{r}, \beta ; \mathbf{r}_{0}, \beta_{0}\right)=\int d \mathbf{r}_{1} \rho\left(\mathbf{r}, \beta ; \mathbf{r}_{1}, \beta_{1}\right) \rho\left(\mathbf{r}_{1}, \beta_{1} ; \mathbf{r}_{0}, \beta_{0}\right) .
$$

Its path integral representation is [1]:

$$
\rho\left(\mathbf{r}_{n}, \beta_{n} ; \mathbf{r}_{0}, \beta_{0}\right)=\int_{\mathbf{r}\left(\beta_{0}\right)}^{\mathbf{r}\left(\beta_{n}\right)} \operatorname{Dr}(\beta) \exp \left[-\frac{m}{2} \int_{\beta_{0}}^{\beta_{n}}\left(\mathbf{r}^{\prime}\right)^{2} d \beta\right],
$$

where $\mathbf{r}^{\prime}=d \mathbf{r} / d \beta$. The partition function $Z(\Delta \beta)$ is defined as:

$$
Z(\Delta \beta)=\left.\int d \mathbf{r} \rho\left(\mathbf{r}, \beta ; \mathbf{r}_{0}, \beta_{0}\right)\right|_{\mathbf{r}(\beta)=\mathbf{r}\left(\beta_{0}\right)} .
$$

In this representation of the density matrix:

a) the states of particles are described in the space-temperature manifold $(\mathbf{r}, \beta)$, i.e. the thermal degree of freedom is treated as a geometrical dimension of the manifold in addition to the spatial and temporal ones; distances along this axis are proportional to the Planck constant $\hbar$ and inverse proportional to the temperature $T$;

b) mixed states for the statistical ensemble of point particles are described by the density matrix $\rho\left(\mathbf{r}, \mathbf{r}_{0} ; \beta\right)$, which after the factorization $\Delta \beta=\beta-\beta_{0}$ may be represented as the transition amplitude $\rho\left(\mathbf{r}, \beta ; \mathbf{r}_{0}, \beta_{0}\right)$ for the "pure" states $\psi_{i}(\mathbf{r}, \beta)$ in the $(\mathbf{r}, \beta)$ - manifold; 
c) the temperature parameter of evolution $\beta$ is unlimited $-\infty \leq \beta \leq \infty$, but the intervals $\Delta \beta$ are restricted as: $0 \leq \Delta \beta \leq 1 / k T$, and therefore, the temperature paths of the particles have finite (mean) lengths;

d) only closed temperature paths with $\mathbf{r}\left(\beta_{n}\right)=\mathbf{r}\left(\beta_{0}\right)$ contribute to the partition function, and we have for their coordinates the periodicity condition $\mathbf{r}(\beta)=\mathbf{r}(\beta+1 / k T)$.

In Feynman's path integral representation of the density matrix [1] there exists the asymmetry between the temporal and thermal degrees of freedom, because of the temperature evolution is described by the path integral, whereas the time evolution is described by the differential equations. Further we shall consider another method for the summation of probabilities for the temperature paths, where both thermal and temporal evolutions are described by the path integrals.

\section{The temperature paths as thermostrings}

The density matrix as the $\beta$-evolution transition amplitude can be represented through infinity set of intermediate states $(n \rightarrow \infty)$ :

$$
\begin{aligned}
\rho\left(\mathbf{r}_{n}, \beta_{n} ; \mathbf{r}_{0}, \beta_{0}\right)= & \int d \mathbf{r}\left(\beta_{1}\right) \ldots d \mathbf{r}\left(\beta_{n-1}\right) \times \\
& \times \rho\left(\mathbf{r}_{n}, \beta_{n} ; \mathbf{r}_{n-1}, \beta_{n-1}\right) \times \ldots \\
& \ldots \times \rho\left(\mathbf{r}_{2}, \beta_{2} ; \mathbf{r}_{1}, \beta_{1}\right) \rho\left(\mathbf{r}_{1}, \beta_{1} ; \mathbf{r}_{0}, \beta_{0}\right),
\end{aligned}
$$

which means that:

$$
\begin{aligned}
\rho\left(\mathbf{r}_{n}, \beta_{n} ; \mathbf{r}_{0}, \beta_{0}\right)= & \int d \mathbf{r}\left(\beta_{1}\right) \ldots d \mathbf{r}\left(\beta_{n-1}\right) \times \\
& \left\{\psi_{i}\left(\mathbf{r}_{n}, \beta_{n}\right) \psi_{i}^{*}\left(\mathbf{r}_{n-1}, \beta_{n-1}\right) \psi_{j}\left(\mathbf{r}_{n-1}, \beta_{n-1}\right) \psi_{j}^{*}\left(\mathbf{r}_{n-2}, \beta_{n-2}\right) \times \ldots\right. \\
& \left.\ldots \times \psi_{k}\left(\mathbf{r}_{2}, \beta_{2}\right) \psi_{k}^{*}\left(\mathbf{r}_{1}, \beta_{1}\right) \psi_{i}\left(\mathbf{r}_{1}, \beta_{1}\right) \psi_{i}^{*}\left(\mathbf{r}_{0}, \beta_{0}\right)\right\} .
\end{aligned}
$$

Here the summation over the repeated indices $i, j, k, \ldots$ is supposed. Due to the normalization conditions, there contribute only the intermediate states with the indices equal to the initial and final states indices: $i=j=k=\ldots$

Therefore, we can transfer all $\psi_{i}\left(\mathbf{r}_{p}, \beta_{p}\right)$ to left hand side and all $\psi_{i}^{*}\left(\mathbf{r}_{p}, \beta_{p}\right)$ to right hand side:

$$
\begin{aligned}
\rho\left(\mathbf{r}_{n}, \beta_{n}, \mathbf{r}_{0}, \beta_{0}\right)= & \int d \mathbf{r}\left(\beta_{1}\right) \ldots d \mathbf{r}\left(\beta_{n-1}\right) \times \\
& \left\{\psi_{i}\left(\mathbf{r}_{n}, \beta_{n}\right) \psi_{i}\left(\mathbf{r}_{n-1}, \beta_{n-1}\right) \ldots \psi_{i}\left(\mathbf{r}_{1}, \beta_{1}\right)\right\} \times \\
& \times\left\{\psi_{i}^{*}\left(\mathbf{r}_{n-1}, \beta_{n-1}\right) \ldots \psi_{i}^{*}\left(\mathbf{r}_{1}, \beta_{1}\right) \psi_{i}^{*}\left(\mathbf{r}_{0}, \beta_{0}\right)\right\},
\end{aligned}
$$

and then rewrite this expression across the wave functionals $\Psi_{i}$ and $\Psi_{i}^{*}$ as:

$$
\rho\left(\mathbf{r}_{n}, \beta_{n} ; \mathbf{r}_{0}, \beta_{0}\right)=\int_{\mathbf{r}\left(\beta_{0}\right)}^{\mathbf{r}\left(\beta_{n}\right)} D \mathbf{r}(\beta) \Psi_{i}\left[\mathbf{r}(\beta) ; \beta_{n}, \beta_{0}\right] \Psi_{i}^{*}\left[\mathbf{r}(\beta) ; \beta_{n}, \beta_{0}\right] .
$$

Here $\operatorname{Dr}(\beta)$ is the path integration measure, the wave functionals $\Psi_{i}$ and $\Psi_{i}^{*}$ describe a state of the temperature path and are composed as the product of infinity number ( $\left.n=\left(\beta_{n}-\beta_{0}\right) / \varepsilon \rightarrow \infty\right)$ intermediate state wave functions $\psi_{i}$ of the same energy $E_{i}$ as: 


$$
\begin{aligned}
& \Psi_{i}\left[\mathbf{r}(\beta) ; \beta, \beta_{0}\right]=\lim _{n \rightarrow \infty} \prod_{k=0}^{n} \psi_{i}\left(\mathbf{r}_{k}, \beta_{k}\right), \\
& \Psi_{i}^{*}\left[\mathbf{r}(\beta) ; \beta, \beta_{0}\right]=\lim _{n \rightarrow \infty} \prod_{k=0}^{n} \psi_{i}^{*}\left(\mathbf{r}_{k}, \beta_{k}\right) .
\end{aligned}
$$

We see that the temperature path of the point particle ( $\beta$ - world line) can be described as some one dimensional physical object in space-temperature manifold with the wave functional $\Psi_{i}$, and further we shall call this object as the thermostring.

In general case the wave functional $\Psi_{i}$ must be symmetrized under the permutations of points of the temperature path (thermostring). These permutations depend on a type of statistics of particles in the Gibbs ensemble, and they determine the type of statistics of the thermostring (bosonic or fermionic). In the ordinary string theory the such permutations impossible since the ordinary strings are introduced as continuous one dimensional objects in the physical space.

\section{The temporal evolution of thermostrings}

During a time interval the temperature path (thermostring) swepts a surface in the space-time-temperature manifold. An action function for the particle at the finite temperature and the temporal evolution of the corresponding density matrix can be described by the summation over all the such surfaces. This circumstance allows us to introduce the thermostring representation of the quantum statistical mechanics of particles or the thermostring quantization of the particle at finite temperatures.

The standard time dependence of the wave functions:

$$
\psi_{i}(\mathbf{r}, \beta, t)=\exp (-i H t) \psi_{i}(\mathbf{r}, \beta),
$$

allows us to obtain a time dependence of the wave functionals $\Psi_{i}$. In $(\mathbf{r}, \beta)$ manifold we have $(d+1)$ - dimensional spacelike coordinates $\mathbf{q}$ with components $(\mathbf{r}, \beta)$. Temporal derivatives of these vectors are space-temperature velocities of the particles, and they can be separated into longitudinal and transverse to the temperature path components:

$$
\frac{\partial \mathbf{q}}{\partial t} \equiv \mathbf{v}=\mathbf{v}_{\perp}+\mathbf{v}_{\mathrm{II}}, \quad \mathbf{v}_{\perp}=\mathbf{v}-\mathbf{k}\left(\mathbf{q}^{\prime} \mathbf{v}\right)
$$

where $\mathbf{k}=\mathbf{q}^{\prime} /\left(\mathbf{q}^{\prime}\right)^{2}$ and $\mathbf{q}^{\prime}=\partial \mathbf{q} / \partial \beta$.

The longitudinal components of the velocity $\mathbf{v}_{\text {II }}$ also have two parts. The first one leads to the collective motion of the thermostring as a whole object with synchronous displacements of all points of the thermostring, and in the case of a single thermostring these displacements can be disregarded (as zero modes). The second part of the longitudinal velocity leads to permutations of neighbor points of the thermostring. These permutations do not contribute to the thermostring energy because of indistinguishability of its points. In the string theory the exclusion of the $\mathbf{v}_{\text {II }}$ from the Lagrangian is one of difficulties of the theory [5], whereas in the case of thermostrings this is a natural and necessary procedure.

So, we have following time dependence formula for the wave functional:

$$
\Psi_{i}\left[\mathbf{q}(\beta, t), \beta_{n}, \beta_{0} ; t\right]=\exp \left(-\frac{i \Delta t}{\Delta \beta} \int_{\beta_{0}}^{\beta_{n}} d \beta H\left(\mathbf{v}_{\perp}^{2}\right)\right) \Psi_{i}\left[\mathbf{q}(\beta) ; \beta_{n}, \beta_{0} ; t_{0}\right] .
$$


This expression can be represented in the form of a surface integral as:

$$
\Psi_{i}\left[\mathbf{q}(\beta, t) ; \Delta \beta ; t_{n}\right]=P[\mathbf{q}(\beta, t) ; \Delta t ; \Delta \beta] \Psi_{i}\left[\mathbf{q}\left(\beta, t_{0}\right) ; \Delta \beta ; t_{0}\right],
$$

where:

$$
\begin{aligned}
P[\mathbf{q}(\beta, t) ; \Delta t ; \Delta \beta] & =\int D \mathbf{q}(\beta, t) \exp \left(\frac{i}{\Delta \beta} \int_{\beta_{0}, t_{0}}^{\beta_{n}, t_{n}} d \beta d t L\left[\mathbf{v}_{\perp}(\beta, t), \mathbf{q}(\beta, t)\right]\right)= \\
& =\int D \mathbf{q}(\beta, t) \exp \{i S[\mathbf{q}(\beta, t) ; \Delta t ; \Delta \beta]\}
\end{aligned}
$$

Here $P[\mathbf{q}]$ is a propagator and $S[\mathbf{q}]$ is the action function for the thermostring:

$$
\begin{aligned}
S[\mathbf{q}] & =\frac{1}{\Delta \beta} \int d \beta d t L\left[\mathbf{v}_{\perp}(\beta, t), \mathbf{q}(\beta, t)\right]= \\
& =\frac{m}{2 \Delta \beta} \int d \beta d t \cdot \mathbf{v}_{\perp}^{2} .
\end{aligned}
$$

For the Gibbs ensemble of free relativistic particles we have following action function for the corresponding relativistic thermostrings:

$$
S[x]=-\frac{m}{\Delta \beta} \int d \beta d t \sqrt{1-\mathbf{v}_{\perp}^{2}},
$$

where $(d+2)$-vector $x^{\mu}$ have components $x^{\mu}(\mathbf{q}, t)=x^{\mu}(\mathbf{r}, \beta, t)$.

As it can be shown [5], after the introduction of the world sheet parameters $\tau, \sigma$ as $t(\tau, \sigma), \beta(\tau, \sigma)$, and the substitutions:

$$
\begin{aligned}
d \beta & =d \sigma \sqrt{x^{\prime 2}}, \\
d t d \sigma & =\frac{\partial(t, \sigma)}{\partial(\tau, \sigma)} d \tau d \sigma=\dot{t} d \tau d \sigma,
\end{aligned}
$$

where $\dot{x}^{\mu}=\partial x^{\mu} / \partial \tau, x^{\prime}=\partial x / \partial \sigma, \partial \mathbf{q} / \partial t=\dot{\mathbf{q}} / \dot{t}$ and $\partial \mathbf{q} / \partial \sigma=\mathbf{q}^{\prime}-\dot{\mathbf{q}} t^{\prime} / \dot{t}$, the action function transforms into the Nambu-Goto action for the relativistic thermostring:

$$
S[x]=-\gamma \int d \sigma d \tau \sqrt{\left(\dot{x} x^{\prime}\right)^{2}-\dot{x}^{2} x^{\prime 2}},
$$

where $\gamma=m / \Delta \beta=m k T$. We see that in the thermostring representation of quantum statistical mechanics of particles there exist the reparametrizational symmetry $\sigma^{\prime}=f(\sigma, \tau), \tau^{\prime}=\phi(\sigma, \tau)$ as in the string theories.

Another form of the action function for the relativistic particle leads to the second form of the action function for the thermostrings [2]:

$$
S[x, e]=-\frac{1}{2 \Delta \beta} \int d \beta d t\left(e^{-1} \mathbf{v}_{\perp}^{2}-e m\right)
$$

This action is fully relativistically invariant if $\Delta \beta$ and the limits of the $\beta$-integration are invariants. In ordinary temperatures it is impossible, but if we take the relativistically invariant (Planck, charge radii, etc.) temperature $T_{0}$ as a limiting temperature for the thermostrings with $\Delta \beta_{0}=1 / k T_{0}$, then we have the invariant action function. In terms of the world-sheet coordinates $x(\sigma, \tau)$ and metrics $g(\sigma, \tau)$ this action leads to the Polyakov surface integral [6] for the thermostring amplitude: 


$$
P[x, g]=\int D x(\sigma, \tau) D g(\sigma, \tau) \exp (i S[x, g])
$$

Finally, we conclude that the statistical mechanics of the relativistic particle at finite temperatures in the thermostring representation is practically identical with the closed string theory formalism.

\section{Statistical mechanics in the thermostring representation}

The thermostring representation may be useful for the solving of equilibrium and non-equilibrium statistical mechanics problems by means of new methods of the string theory formalism. The surface integral representation of the Green functions of statistical mechanics and the geometric formulation can lead to some nontrivial consequences.

As an example can be considered the problem of doubling of operators in the thermofield dynamics, which developed by H.Umezawa et al. [7]. In the thermostring representation this doubling can be explained as the consequence of the existence of left and right moving modes for the closed thermostrings. The thermal algebra for these doubled states can be represented as the Virasoro algebra for the thermostring modes.

Another nontrivial consequence of the thermostring quantization may be the appearance of the Liouville modes and tachyons as additional fields or collective excitations for some statistical systems. The Liouville modes of the thermostrings in statistical mechanics appear because of the dimensionality of the manifold is less than the critical dimensionality of the manifold (10 or 26). Tachyons also may appear in the thermostring quantization of some statistical systems due to the absence of fermions or supersymmetry [2].

\section{Thermostring interpretation of closed strings}

In the standard string theory the strings have been introduced as new fundamental one dimensional objects in the physical space. The introduction into physics of the such non-local structures leads to conceptual difficulties concerning measurability of points of the strings, their intrinsic dynamics, relativistic causality, etc. There is a disconnection between the pointness of the space-time manifold with metrics and non-local nature of the strings, some modes of which generate gravitons. Strings and other their generalizations, such as branes, are alien to the geometry of space-time and the reduction of the strings and branes to space-time properties is nontrivial problem.

The thermostring quantization does not introduce new objects into physics, the dimensionality of the space is lower to one (with respect to the string theories), it practically coincides with the string theory formalism, and it can be used directly for the description of the physical systems without any hypothesis. This is only a new method of quantization at finite temperatures of the ordinary objects - point particles and local fields, where the thermostring appears as some non-local structure in the configurational space-time-temperature manifold. A physical reason for non-locality is the Gibbs ensemble averaging and the thermostring length is nothing but as the instantaneous length of the space-temperature world-line of the point particle. So, in the thermostring representation we introduce into physics only a new (temperature) dimension in addition to the space and time, and we represent the physical manifold as the space-timetemperature manifold with more general properties than in space-time.

The superstrings [2] can be interpreted as thermostrings due to a practical identity of the formalisms by the preserving all achievements of the string theory. At the same time, the new treatment allows one to exclude some conceptual difficulties associated with the introduction into physics of non-local fundamental objects with immeasurable intrinsic structure. 
Thermostring interpretation of the superstrings leads to the following consequences:

a) strings are not fundamental physical objects in the physical space, they are point particles moving in the fluctuating background with some invariant temperature. They are described by the string formalism because of the need for Gibbs ensemble averaging;

b) one of dimensions of the string theory manifold is the thermal dimension, and, therefore, the dimensionality of the space is equal to 8 (or 24), which, together with temporal and thermal degrees of freedom, form the critical dimensionality;

c) only the closed strings appear at the initial and final states of the string theory amplitudes as observable physical states;

d) the charge of the particle must be distributed along the thermostring;

e) the interactions of the strings with point particles become more clearly understandable;

f) at large distances (and low temperatures) the invariant temperature $T_{0}$ can be considered as a very large quantity $T_{0} \rightarrow \infty$, and the thermostrings with $\Delta \beta \rightarrow 0$ becomes like to a point particle.

Among superstring theories only theories of closed strings satisfy to these conditions, and we may conclude that only the superstrings IIA (in the case of neutral particles) and the heterotic strings (in the case of charged particles) can be interpreted as thermostrings.

The thermostring interactions may be described simultaneously in the particle, statistical ensemble and string languages. The factorization of one a statistical ensemble into two subensembles, or the merging of two ensembles into one general are physically clearer and simpler procedures than cutting or gluing of rigid strings in the string theory.

We can perform the thermostring quantization of physical strings as one dimensional objects in physical space and we shall obtain as a result the theory of thermomembranes. So, if we provide the thermostring quantization of the physical D-branes, we shall obtain the theory of (D+1)-thermobranes, i.e. a dimensionality of initial objects of the theory increases to one. In the treatment of the D-branes the thermostring representation can be combined with M-Theory methods if we interpret one of it's 11 dimensions as temperature degree of freedom.

Finally, we conclude that the thermostring is not only simplest and natural, but necessary modification of the local theories at small distances and high temperatures.

\section{References}

1. Feynman R.P., Hibbs A.R. (1965) Quantum Mechanics and Path Integrals. McG-H.

2. Green M.B., Schwarz J.H., Witten E. (1987) Superstring Theory, Cambridge U.Press.

3. Zakir Z. (2010) Theor. Phys., Astrophys. \& Cosmol., 5, 1, 8; arXiv:hep-th/9810247, 5 p.

4. Zakir Z.(Israilov) (1992) On the Statistical Interpretation of String Theories. In Proc.Int.Conf.Quantum Physics and The Universe, Tokyo, Aug.19-22, 1992; Vistas in Astronomy (1993) v.37 (1-4), p. 277.

5. Barbashov B.M., Nesterenko V.V. (1987) Model of Relativistic String in Hadron Physics (in Russian), Nauka, M.

6. Polyakov A.M. (1981) Phys.Lett. v.103B, pp.207, 211.

7. Umezawa, H. Matsumoto and M. Tachiki, Thermo Field Dynamics and Condensed States, North-Holland 1982. 